www.nature.com/pj

\title{
Preparation of hemispherical particles by cleavage of micrometer-sized, spherical polystyrene/poly(methyl methacrylate) composite particles with Janus structures: effect of polystyrene- $b$-poly(methyl methacrylate)
}

\begin{abstract}
Nobuko Yamashita ${ }^{1}$ and Masayoshi Okubo ${ }^{1,2,3}$
We recently reported that two micrometer-sized, hemispherical polymer particles were prepared as a result of the cleavage of spherical Janus polystyrene (PS)/poly(methyl methacrylate) (PMMA) composite particles after treatment with acetone. The original composite particles were prepared by slow evaporation of toluene from homogeneous PS/PMMA/toluene droplets dispersed in an aqueous solution of sodium dodecyl sulfate. This article aims to validate a proposed cleavage mechanism which is based on the induction of internal stress at the interface because of a great difference in the swelling ratio between the PS and PMMA phases. The effect of PS- $b$-PMMA, which should exist at the interface between both phases and decrease the internal stress, on the cleavage phenomenon was examined. PS/PS- $b$-PMMA/PMMA composite particles were prepared in the same way as the previous study. At $0.31 \mathrm{wt} \%$ or greater of PS- $b$-PMMA (number-average molecular weight: $10.6 \times 10^{4}-b$ $9.9 \times 10^{4}$ ), the composite particles did not have a Janus structure but an ex-centered core-shell structure, resulting in no cleavage. At 0.18 wt $\%$ or less, they had a Janus structure, and the cleavage phenomenon was observed. The required time to cleave increased with increasing PS- $b$-PMMA content to below $0.18 w t \%$. Specifically, the existence of PS- $b$-PMMA delayed the cleavage of the composite particles into PS and PMMA hemispherical particles. This result supports the proposed cleavage mechanism and provides a tool to easily address the cleavage process for the production of hemispherical particles.
\end{abstract}

Polymer Journal (2015) 47, 255-258; doi:10.1038/pj.2014.95; published online 26 November 2014

\section{INTRODUCTION}

Recently, we found that spherical Janus polystyrene (PS)/poly(methyl methacrylate) (PMMA) composite particles were cleaved at the interface between the PS and PMMA phases into two parts (hemispherical particles) in seconds by making contact with acetone. ${ }^{1}$ The original PS/PMMA composite particles were prepared by the slow evaporation of toluene as a common good solvent from toluene droplets, in which PS was dissolved and included PMMA that was dispersed in aqueous solutions of various surfactants (solvent evaporation method)..$^{2-4}$ The particle morphology was formed by not kinetic but by thermodynamic control.,

To our knowledge, such a cleavage phenomenon had not been reported and will become a novel method for the preparation of nonspherical particles, which is one of the functional applications of polymer particles. We proposed a possible formation mechanism of such hemispherical particles as follows. First, the higher swelling of the PMMA phase compared with PS phase with acetone causes stress at the interface between the two phases. The partial dissolution of the PMMA surface layer in contact with water reduces the interfacial area between the two phases. The swelling of the PS and PMMA phases decreases the entanglement between the PS and PMMA molecules at the interface. When the stress caused at the interfaces overcomes the interfacial interaction, the composite particles are rapidly cleaved to PS and PMMA hemispherical particles. Therefore, control of the interaction and reduction of the internal stress at the interface between PS and PMMA should be significant to prepare such hemispherical particles. In a previous article, the effect of the molecular weight of PS and PMMA on the control of the interaction was examined. An increase in the molecular weights of PS and PMMA increased the time required to cleave $\left(T_{\mathrm{c}}\right)$ the spherical Janus PS/PMMA composite particles, which seemed to be because of the difference in the swelling ratios and rates of both the phases, and there was an appropriate molecular weight for the cleavage. ${ }^{7}$ In contrast, there are many studies regarding the existence of block polymers at the interface between two

${ }^{1}$ Department of Chemical Science and Engineering, Graduate School of Engineering, Kobe University, Kobe, Japan; ${ }^{2}$ Department of Chemistry, Faculty of Science and Technology, Rajamangala University of Technology, Thanyaburi, Thailand and ${ }^{3}$ Smart Spheres Workshop Co., Ltd., Kobe, Japan

Correspondence: Professor M Okubo, Graduate School of Engineering, Kobe University, Rokko, Kobe 657-8501, Japan.

E-mail: okubo@kobe-u.ac.jp

Part CCCLX of the series "Studies on Suspension and Emulsion."

Received 16 June 2014; revised 12 September 2014; accepted 15 September 2014; published online 26 November 2014 
different homopolymer phases that have low compatibility with each other, which should decrease the internal stress by creating a smooth transmission because of a gradual modulus gradient at the interfacial layer. ${ }^{8,9}$ We succeeded in preparing 'onion-like' multilayered PS/PS- $b$ PMMA/PMMA composite particles using the solvent evaporation method, in which PS- $b$-PMMA should exist at the interface between the PS and PMMA phases and decrease the interfacial tension. ${ }^{10}$

In this study, to clarify the cleavage mechanism in greater detail, the effect of PS- $b$-PMMA on the cleavage of Janus PS/PMMA composite particles will be examined.

\section{EXPERIMENTAL PROCEDURE}

\section{Materials}

All of the materials used in this study, except PS- $b$-PMMA, were the same as those in the previous article. ${ }^{7}$

\section{Preparation of Janus PS/PS- $b$-PMMA/PMMA composite particles} PS and PMMA were synthesized by solution polymerizations of styrene and methyl methacrylate, respectively, with $2,2^{\prime}$-azobis(isobutyronitrile) in toluene at $70^{\circ} \mathrm{C}$ for $24 \mathrm{~h}$. The prepared polymers were purified by a reprecipitation method using toluene/methanol. The weight- and number-average molecular weights ( $M \mathrm{w}$ and $M \mathrm{n}$, respectively) were measured using gel permeation chromatography. The molecular weights of PS, PMMA and PS- $b$-PMMA are shown in Table 1. Different molecular weights of PS- $b$-PMMA were purchased from Polymer Source Inc. (Dorval, QC, Canada).

Homogeneous toluene solutions (0.41 g) of PS, PMMA and PS- $b$-PMMA $(\mathrm{PS} / \mathrm{PMMA} /$ toluene $=1: 1: 23(\mathrm{w} / \mathrm{w} / \mathrm{w}))$ were prepared. Various amounts of PS$b$-PMMA (as shown in Table 2) were mixed with an aqueous solution (9.2 g) of sodium dodecyl sulfate (SDS) $(63.6 \mathrm{~mm})$, and the mixtures were stirred vigorously using a homogenizer (Nihonseiki Kaisya Ltd., AMB-2, Tokyo, Japan) at 4000 r.p.m. for $3 \mathrm{~min}$ in a $30-\mathrm{ml}$ glass vial. The prepared toluene droplets were polydisperse. The toluene was slowly evaporated from the dispersions under gentle stirring with a magnetic stirrer at room temperature for $24 \mathrm{~h}$ in uncovered glass vials (the interfacial area between air and the dispersion: $6 \mathrm{~cm}^{2}$ ). The obtained particles dispersed in the sodium dodecyl sulfate aqueous solutions were repeatedly washed by serum replacement with water. The Janus PS/PS-b-PMMA/PMMA composite particles were observed using an optical microscope (Nikon Corp., MICROPHOT-FXA, Tokyo, Japan).

\section{Observation of the cleavage of the Janus particles}

A homogeneous solution of acetone/water was dropped onto dried particles on a microscope slide for observation using an optical microscope (Nikon Corp., MICROPHOT-FXA, Tokyo, Japan) equipped with a high-speed camera (Photron Ltd., FASTCAM-512PCI, Tokyo, Japan). The time required to cleave the particles (cleavage time, $T_{\mathrm{c}}$ ) after making contact with the solution was measured for composite particles having different diameters.

\section{RESULTS AND DISCUSSION}

As shown in the optical micrographs in Figure 1, the PS/PMMA (1/1, $\mathrm{w} / \mathrm{w})$ composite particles including PS- $b$-PMMA at $0.18 \mathrm{wt} \%$ or less content had a Janus structure. However, the particles including PS- $b$ PMMA at $0.31 \mathrm{wt} \%$ or greater did not have the Janus structure. Instead, they had an ex-centered core-shell structure (micrograph e), which seemed to be due to a decrease in the interfacial tension at the interface between the PS and PMMA phases due to the increase in the PS- $b$-PMMA content. Therefore, the effect of PS- $b$-PMMA on the cleavage of PS/PMMA composite particles was examined using the composite particles including $0.18 \mathrm{wt} \%$ or less of PS- $b$-PMMA.

Figure 2 shows the $T_{\mathrm{c}}$ values for PS/PMMA composite particles with different PS- $b$-PMMA (B11) content as a function of the interfacial area of the two phases that was calculated from the diameter. The $T_{\mathrm{c}}$ value increased linearly as the interfacial area increased and had a tendency to increase as the PS- $b$-PMMA content increased, although
Table 1 Weight- and number-average molecular weights ( $M w$ and $M n$, respectively) of PS, PMMA and PS- $b$-PMMA

\begin{tabular}{lccc}
\hline & $M_{\text {PS }}\left(\times 10^{4}\right)^{\mathrm{a}}$ & $M_{\text {PMMA }}\left(\times 10^{4}\right)^{\mathrm{b}}$ & $M w / M n$ \\
\hline PSc $^{c}$ & 5.7 & 5.8 & 1.7 \\
PMMAc $^{c}$ & & 1.9 \\
PS- $b-P M M A^{d}$ & & 1.0 & \\
B1 & 1.0 & 9.9 & 1.1 \\
B11 & 10.6 & 28.9 & 1.1 \\
B27 & 27.0 & & 1.2 \\
\hline
\end{tabular}

Abbreviations: AIBN, 2,2'-azobis(isobutyronitrile); PMMA, poly(methyl methacrylate); PS, polystyrene.

${ }^{a} M n$ of PS or PS segment in PS- $b$-PMMA

$\mathrm{M}$ n of PMMA or PMMA segment in PS- $b$-PMMA.

'Prepared by solution polymerization in toluene with AIBN.

dPurchased from Polymer Source Inc., Canada.

Table 2 Recipes for the production of PS/PMMA (1/1, w/w) composite particles including different amounts of PS- $b$-PMMA using the solvent evaporation method

\begin{tabular}{|c|c|c|c|c|c|}
\hline \multirow{2}{*}{ Ingredients } & \multicolumn{5}{|c|}{$P S-b-P M M A$ content (wt \%) } \\
\hline & 0 & 0.05 & 0.11 & 0.18 & 0.31 \\
\hline $\mathrm{PS}^{\mathrm{a}}(\mathrm{mg})$ & 16.5 & 16.5 & 16.5 & 16.5 & 16.5 \\
\hline $\mathrm{PMMA}^{\mathrm{b}}$ (mg) & 16.5 & 16.5 & 16.5 & 16.5 & 16.5 \\
\hline PS- $b-\mathrm{PMMA}^{\mathrm{c}}(\mathrm{mg})$ & 0 & 0.018 & 0.036 & 0.059 & 0.102 \\
\hline Toluene (mg) & 375 & 375 & 375 & 375 & 375 \\
\hline SDS (mg) & 165 & 165 & 165 & 165 & 165 \\
\hline Water (g) & 9 & 9 & 9 & 9 & 9 \\
\hline
\end{tabular}

Abbreviations: PMMA, poly(methyl methacrylate); PS, polystyrene; SDS, sodium dodecyl sulfate. Dispersion of polymer droplets: stirred by a homogenizer at 4000 r.p.m for $3 \mathrm{~min}$ Release of toluene: stirred with a magnetic stirrer at room temperature for $24 \mathrm{~h}$ in the uncovered glass vials (interfacial area between air and dispersion: $6 \mathrm{~cm}^{2}$ ).

a $M n: 5.7 \times 10^{4}, M_{w} / M n: 1.7$

${ }^{\mathrm{b}} \mathrm{Mn}: 5.8 \times 10^{4}, M \mathrm{w} / \mathrm{Mn}: 1.9$

'B11 in Table 1; $M \mathrm{n}: 10.6 \times 10^{4}-b-9.9 \times 10^{4}, M \mathrm{w} / \mathrm{Mn}: 1.1$

the data were slightly scattered. This result indicates that PS- $b$-PMMA suppressed the cleavage of the PS/PMMA composite particles. Specifically, PS- $b$-PMMA might increase the interfacial interaction between PS and PMMA phases in the composite particles.

Figure 3 shows the relationships between the PS- $b$-PMMA molecules with different molecular weights and the $T_{c}-T_{\mathrm{c}}{ }^{\mathrm{o}}$ values $\left(T_{\mathrm{c}}{ }^{\mathrm{o}}\right.$ is $T_{\mathrm{c}}$ in a PS-b-PMMA-free system). In the systems using the lowest (B1) and the highest (B27) molecular weights, Janus particles were not obtained when the PS- $b$-PMMA content relative to the total amount of PS and PMMA was increased beyond threshold values. This result is similar to that using an intermediate molecular weight (B11), as previously described above. Therefore, the effect of the PS- $b$-PMMA content on the $T_{\mathrm{c}}-T_{\mathrm{c}}{ }^{\mathrm{O}}$ value was investigated below the threshold values, at which the PS/PMMA composite particles had Janus structures. In the case of $\mathrm{B} 1\left(\mathrm{Mn}: 1.0 \times 10^{4}-b-1.0 \times 10^{4}\right)$, there was little change in the $T_{\mathrm{c}}-T_{\mathrm{C}}{ }^{\mathrm{o}}$ value. In the case of B11 (Mn: $10.6 \times 10^{4}-b$ $\left.9.9 \times 10^{4}\right)$, the $T_{\mathrm{c}}-T_{\mathrm{c}}{ }^{\mathrm{o}}$ value increased with the PS- $b$-PMMA content. These results suggest that there is a minimum block chain length to decrease the interface stress. However, the $T_{\mathrm{c}}$ was shorter in B27 $(\mathrm{Mn}$ $\left.27 \times 10^{4}-b-28.9 \times 10^{4}\right)$ compared with $\mathrm{B} 11$. This result might be due to decreases in the number and mobility of PS- $b$-PMMA molecules in the particles as a result of increasing the molecular weight, which 

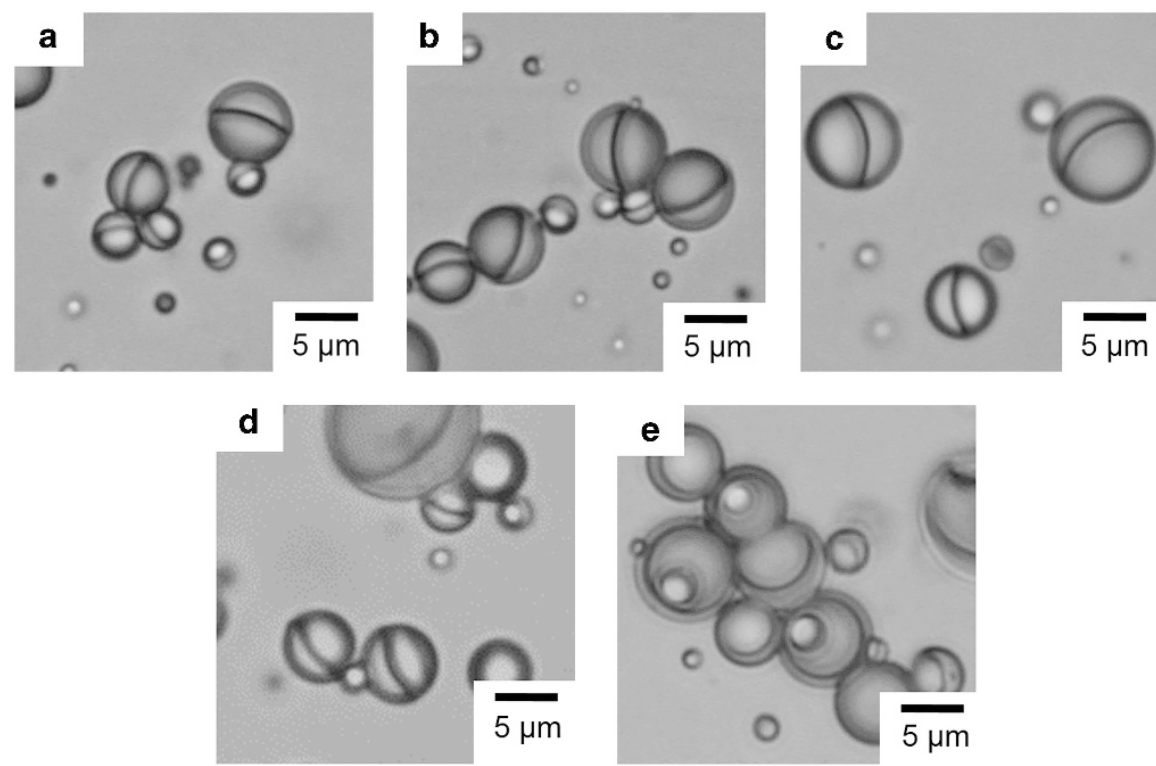

Figure 1 Optical micrographs of polystyrene/poly(methyl methacrylate) $(1 / 1, w / w)$ composite particles including various amounts of polstyrene- $b$-poly(methyl methacrylate) ((a) 0 ; (b) 0.05 ; (c) 0.11 ; (d) 0.18 ; and (e) 0.31 wt\% relative to the total amount of polystyrene and poly(methyl methacrylate)) prepared by the slow evaporation of toluene at room temperature for $24 \mathrm{~h}$ from polymer/toluene $(1 / 12, \mathrm{w} / \mathrm{w})$ droplets dispersed in aqueous solution of sodium dodecyl sulfate.

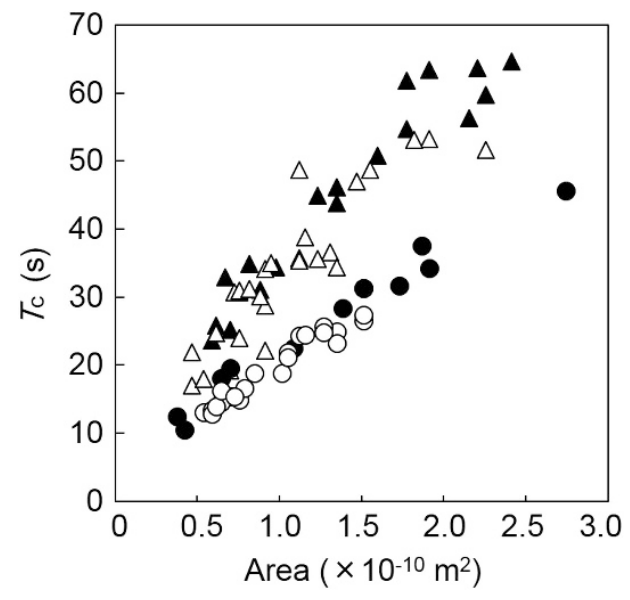

Figure 2 Relationships between the interfacial area between polystyrene and poly(methyl methacrylate) in polystyrene/poly(methyl methacrylate) $(1 / 1$, w/w) composite particles including various amounts (white circles 0; filled circles 0.05 ; white triangles 0.11 ; filled triangles $0.18 \mathrm{wt} \%$ relative to the total amount of polystyrene and poly(methyl methacrylate)) of polystyrene- $b$-poly (methyl methacrylate) (B11: $\left.M n 10.6 \times 10^{4}-b-9.9 \times 10^{4}\right)$, and the required time to cleave into two parts (cleavage time, $T_{\mathrm{c}}$ ) by the addition of an acetone/water $(9: 1, \mathrm{v} / \mathrm{v})$ solution to the dried particles.

results in low number of PS- $b$-PMMA molecules at the interface between the polymer phases.

\section{CONCLUSIONS}

PS-b-PMMA affected the cleavage phenomenon of spherical Janus PS/PMMA composite particles caused by contact with an acetone/ water $(9: 1, \mathrm{v} / \mathrm{v})$ solution, which resulted in hemispherical PS and PMMA particles. Specifically, the existence of PS- $b$-PMMA increased the $T_{\mathrm{c}}$. The molecular weight of PS- $b$-PMMA also affected the

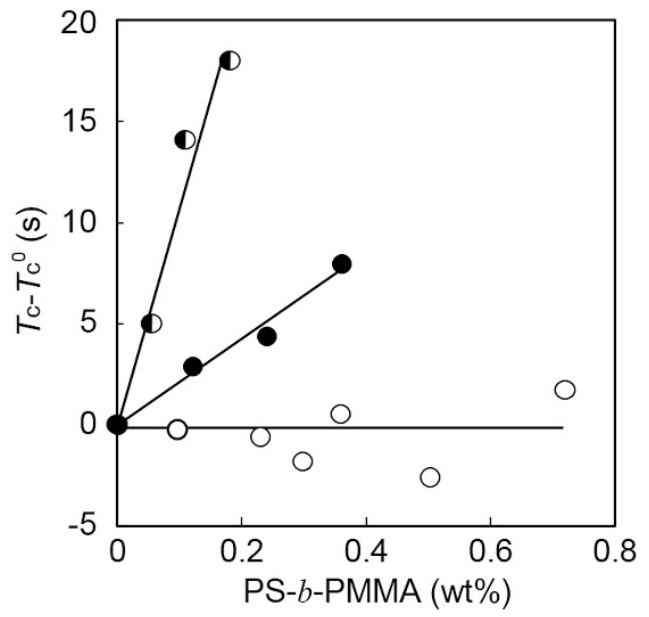

Figure 3 Relationships between the content of polystyrene- $b$-poly(methyl methacrylate) with various $\mathrm{Mn}$ values (white circles B1: $1.0 \times 10^{4}-b$ $1.0 \times 10^{4}$; half circles B11: $10.6 \times 10^{4}-b-9.9 \times 10^{4}$; filled circles B27: $\left.27.0 \times 10^{4}-b-28.9 \times 10^{4}\right)$ relative to the total amount of polystyrene and poly (methyl methacrylate) and the $T_{c}-T_{c}{ }^{\circ}$ in the cleavage process with an acetone/water $(9: 1, \mathrm{v} / \mathrm{v})$ solution. $T_{\mathrm{c}}{ }^{0}$ is $T_{\mathrm{c}}$ in a polystyrene- $b$-poly(methyl methacrylate)-free system.

cleavage. These results strongly support the previously proposed cleavage mechanism of spherical PS/PMMA composite particles with Janus structures by selective solvent absorption. The effect of PS- $b$ PMMA, which should localize at the interface between the PS and PMMA phases, on the interfacial stress was visualized by the cleavage phenomenon. As shown in the previous article, ${ }^{7}$ the hemispherical particles rapidly became spherical. Therefore, controlling the cleavage time $\left(T_{c}\right)$ is important for the production of hemispherical particles, which may be achieved using PS- $b$-PMMA. 


\section{ACKNOWLEDGEMENTS}

This work was partially supported by the Support Program from a Grant-inAid for Scientific Research (Grant 25288054) from the Japan Society for the Promotion of Science (JSPS).

1 Yamashita, N., Konishi, N., Tanaka, T. \& Okubo, M. Preparation of hemispherical polymer particles by cleavage of a Janus poly(methyl methacrylate)/polystyrene composite particle. Langmuir 28, 12886-12892 (2012).

2 Okubo, M., Saito, N. \& Fujibayashi, T. Preparation of polystyrene/poly(methy methacrylate) composite particles having a dent. Colloid. Polym. Sci. 283 691-698 (2005).

3 Saito, N., Kagari, Y. \& Okubo, M. Effect of colloidal stabilizer on the shape of polystyrene/poly(methyl methacrylate) composite particles prepared in aqueous medium by the solvent evaporation method. Langmuir 22, 9397-9402 (2006).
4 Tanaka, T., Nakatsuru, R., Kagari, Y., Saito, N. \& Okubo, M. Effect of molecular weight on the morphology of polystyrene/poly(methyl methacrylate) composite particles prepared by the solvent evaporation method. Langmuir 24, 12267-12271 (2008)

5 Okubo, M. Control of particle morphology in emulsion polymerization. Makromol. Chem. Macromol. Symp. 35/36, 307-325 (1990).

6 Sundberg, D. C. \& Durant, Y. G. Latex particle morphology, fundamental aspects: A review. Polym. React. Eng. 11, 379-432 (2003).

7 Yamashita, N., Yamagami, T. \& Okubo, M. Preparation of hemispherical particles by cleavage of micrometer-sized, spherical poly(methyl methacrylate)/polystyrene composite particle with Janus structure: effect of molecular weight. Colloid. Polym. Sci. 292, 733-738 (2014)

8 Creton, C., Hui, C., Brown, H. R. \& Kramer, E. J. Failure mechanisms of polymer interfaces reinforced with block copolymers. Macromolecules 25, 3075-3088 (1992).

9 Washiyama, J., Hui, C. \& Kramer, E. J. Fracture mechanisms of polymer interfaces reinforced with block copolymers - transition from chain pullout to crazing. Macromolecules 26, 2928-2934 (1993).

10 Okubo, M., Saito, N., Takekoh, R. \& Kobayashi, H. Morphology of polystyrene/ polystyrene-block-poly(methyl methacrylate)/poly(methyl methacrylate) composite particles. Polymer 46, 1151-1156 (2005) 